\title{
recillunds
}

Revista Cientifica Mundo de la Investigación y el Conocimiento

\author{
Becquer Humberto Suarez Coba ${ }^{\text {a; }}$ Patricia Elisabeth Borja Tapia ${ }^{\text {b; }}$ Mayra
}

Alexandra Vela Chasiluisa ${ }^{\mathrm{c}}$; Carlos Fabián Ontaneda Tenesaca ${ }^{\mathrm{d}}$

Diagnóstico y manejo del síndrome de ovario poliquístico

Diagnosis and management of the poliquistic ovary syndrome

Revista Científica Mundo de la Investigación y el Conocimiento. Vol. 3 núm.3,

septiembre, ISSN: 2588-073X, 2019, pp. 970-1004

DOI: $10.26820 /$ recimundo/3.(3).septiembre.2019.970-1004

URL: http://recimundo.com/index.php/es/article/view/557

Código UNESCO: 3205 Medicina Interna

Tipo de Investigación: Artículo de Revisión

Editorial Saberes del Conocimiento

Recibido: $15 / 05 / 2019 \quad$ Aceptado: 23/06/2019 Publicado: 30/09/2019

Correspondencia: bec.suarez70@gmail.com

a. Especialista en Atención Primaria de la Salud; Especialista en Gerencia y Planificación Estratégica de Salud; Especialista en Medicina Familiar y Comunitaria; Doctor en Medicina y Cirugía; Investigador Independiente; Guayaquil, Ecuador; bec.suarez70@gmail.com

b. Magister en Seguridad y Prevención de Riesgos del Trabajo; Diploma Superior en Desarrollo Local y Salud; Especialista en Medicina Familiar y Comunitaria; Doctor en Medicina y Cirugía; Investigador Independiente; Guayaquil, Ecuador; patricia-borja@ hotmail.com

c. Magister en Salud Publica; Especialista en Gestión de Proyectos en Salud; Diploma Superior en Promoción y Prevención de la Salud; Especialista en Medicina Familiar y Comunitaria; Doctora en Medicina y Cirugía; Investigador Independiente; Guayaquil, Ecuador; dramayravelach@ hotmail.com

d. Especialista de Primer Grado en Medicina General Integral; Doctor en Medicina; Investigador Independiente; Guayaquil, Ecuador; carlosontaneda_20@yahoo.es 


\section{Diagnóstico y manejo del síndrome de ovario poliquístico}

Vol. 3, núm. 3., (2019)

Becquer Humberto Suarez Coba; Patricia Elisabeth Borja Tapia; Mayra Alexandra Vela

Chasiluisa; Carlos Fabián Ontaneda Tenesaca

\section{RESUMEN}

El síndrome de ovario poliquístico (SOP) es un trastorno endocrino metabólico altamente prevalente y se considera en la actualidad una patología familiar. Su presencia debe sospecharse en cualquier adolescente o mujer en edad reproductiva con hirsutismo u otras manifestaciones cutáneas de hiperandrogenismo, irregularidades menstruales y obesidad. El síndrome de ovario poliquístico es un diagnóstico de exclusión y por lo tanto, debe diferenciarse de los cambios fisiológicos propios de la edad y de otros trastornos hiperandrogénicos que requieren de una terapia específica. El diagnóstico oportuno es importante ya que este síndrome se asocia a riesgos reproductivos, oncológicos y metabólicos. El abordaje diagnóstico implica ciertas dificultades en relación al momento de presentación de los síntomas y la tendencia actual es considerarlo como un diagnóstico de exclusión, empleándose los cuatros fenotipos propuestos en Rotterdam, lo que implica que estas pacientes deben ser diagnosticadas y tratadas oportunamente, necesitan ser informadas y educadas sobre su patología. El tratamiento de esta enfermedad debe iniciarse siempre con la corrección de las alteraciones metabólicas, la aparición de sus síntomas puede estar condicionada por el medio ambiente y la herencia genética actuando sobre etapas tempranas de la vida. Existen varios fenotipos de SOP, entre los que se encuentran: los criterios de Rotterdam (2003): Obesidad con hiperandrogenismo y poliquistosis, poliquistosis y obesidad sin hirsutismo clínico, obesidad e hirsutismo sin poliquistosis asociada, y finalmente poliquistosis e hiperandrogenismo sin obesidad, por orden de prevalencia. Existen numerosas complicaciones asociadas a esta entidad, como pueden ser cambios en el humor, hígado graso no alcohólico, aumento de riesgo cardiovascular, aumento del riesgo del cáncer de endometrio y otros. En los últimos años se ha podido establecer, que este trastorno no sólo está limitado a la mujer en etapa reproductiva, sino que puede manifestarse desde el período prepuberal y quizás antes.

Palabras Claves: Abordaje Diagnóstico; Ovario Poliquístico; Hiperandrogenismo; Irregularidades Menstruales; Obesidad; Consenso de ROTTERDAM. 


\title{
Diagnóstico y manejo del síndrome de ovario poliquístico
}

Vol. 3, núm. 3., (2019)

Becquer Humberto Suarez Coba; Patricia Elisabeth Borja Tapia; Mayra Alexandra Vela Chasiluisa; Carlos Fabián Ontaneda Tenesaca

\begin{abstract}
Polycystic ovary syndrome (PCOS) is a highly prevalent metabolic endocrine disorder and is currently considered a family pathology. Its presence should be suspected in any adolescent or woman of reproductive age with hirsutism or other cutaneous manifestations of hyperandrogenism, menstrual irregularities and obesity. Polycystic ovarian syndrome is a diagnosis of exclusion and therefore, it must be distinguished from the physiological changes of age and other hyperandrogenic disorders that require specific therapy. Timely diagnosis is important since this syndrome is associated with reproductive, oncological and metabolic risks. The diagnostic approach implies certain difficulties in relation to the time of presentation of the symptoms and the current tendency is to consider it as a diagnosis of exclusion, using the four phenotypes proposed in Rotterdam, which implies that these patients should be diagnosed and treated in a timely manner, they need to be informed and educated about their pathology. The treatment of this disease should always begin with the correction of metabolic alterations, the appearance of its symptoms may be conditioned by the environment and genetic inheritance acting on early stages of life. There are several PCOS phenotypes, among which are: the Rotterdam criteria (2003): Obesity with hyperandrogenism and polycystic disease, polycystic disease and obesity without clinical hirsutism, obesity and hirsutism without associated polycystic disease, and finally polycystosis and hyperandrogenism without obesity, in order of prevalence. There are numerous complications associated with this entity, such as changes in mood, non-alcoholic fatty liver, increased cardiovascular risk, increased risk of endometrial cancer and others. In recent years it has been established that this disorder is not only limited to women in the reproductive stage, but can manifest itself from the prepubertal period and perhaps earlier.
\end{abstract}

Key Words: Diagnostic Approach; Polycystic Ovary; Hyperandrogenism; Menstrual Irregularities; Obesity; ROTTERDAM Consensus. 


\section{Diagnóstico y manejo del síndrome de ovario poliquístico}

Vol. 3, núm. 3., (2019)

Becquer Humberto Suarez Coba; Patricia Elisabeth Borja Tapia; Mayra Alexandra Vela

Chasiluisa; Carlos Fabián Ontaneda Tenesaca

\section{Introducción.}

En 1935, Stein y Leventhal denominaron síndrome de ovarios poliquísticos (SOP) a un cuadro clínico caracterizado por la presencia de ovarios con pequeños quistes, amenorrea, hirsutismo y obesidad. Inicialmente fue llamado síndrome de Stein Leventhal, y en la actualidad como hiperandrogenismo ovárico funcional. (Calletano, 2016). Esta patología, representa un grave problema de salud pública, y la más común de los desórdenes endocrinos en mujeres en edad reproductiva, puesto que es heterogénea y se encuentra caracterizada por anovulación crónica hiperandrogénica, secundaria a una disfunción ovárica intrínseca y tiene un fuerte componente genético.

El Síndrome de Ovario Poliquístico, afecta a un 4-10 \% de la población femenina en edad fértil; pero se ha hallado ovario poliquístico $(\mathrm{OP})$ en $24 \%$ de las niñas entre 3 y 18 años. Las adolescentes que acuden a consulta por trastornos menstruales, hirsutismo (presente hasta en el $80 \%$ de los casos), obesidad o la presencia ultrasonográfica accidental de SOP, muchas veces son valoradas por separado entre endocrinólogos, ginecólogos o internistas sin la debida visión integral de riesgo vascular, en respuesta a la insulinoresistencia (IR) piedra angular del síndrome metabólico (SM) y presente también en el SOP. Por tal razón, a pesar del tiempo transcurrido desde la primera descripción del SOP por Stein y Leventhal, aún es motivo de controversia cuáles son los elementos clínicos, hormonales y ecográficos que mejor definen la entidad. (Calletano, 2016)

Imagen $\mathbf{N}^{\circ}$ 1. Síndrome de Ovario Poliquístico 


\section{Diagnóstico y manejo del síndrome de ovario poliquístico}

Vol. 3, núm. 3., (2019)

Becquer Humberto Suarez Coba; Patricia Elisabeth Borja Tapia; Mayra Alexandra Vela Chasiluisa; Carlos Fabián Ontaneda Tenesaca

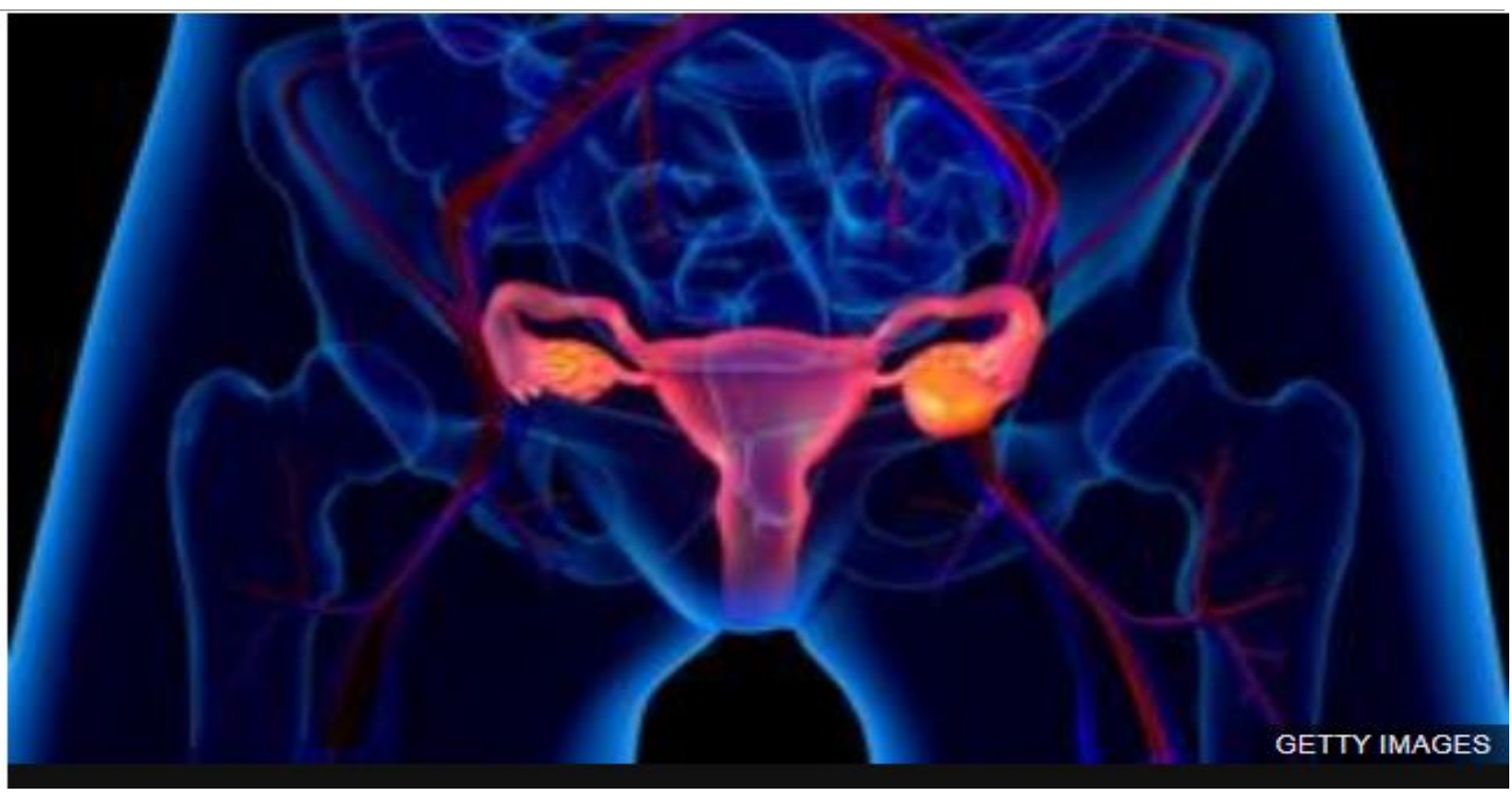

Fuente: (Calletano, 2016).

Para el año de 1990, el National Institutes of Health (NIH) lo definió como la "presencia de hiperandrogenismo asociado a anovulación crónica sin otra causa específica de enfermedad adrenal o hipofisiaria que curse con irregularidades menstruales o exceso de andrógenos", sin considerar el aspecto morfológico de los ovarios. (Pereira, 2016). Posterior a ello, la Sociedad Europea de Reproducción y Embriología (ESHRE) y la Sociedad Americana de Medicina Reproductiva (ASRM) en Rotterdam (2003), propuso una nueva definición del síndrome: oligovulación y/o anovulación, hiperandrogenismo clínico y/o bioquímico e incorporó la presencia de morfología de ovarios poliquísticos (MOP) a través de la ultrasonografía.

En términos generales, el síndrome de ovario poliquístico (SOP) es y ha representado un trastorno endocrino metabólico altamente prevalente y se considera en la actualidad una patología familiar. Su presencia debe sospecharse en cualquier adolescente o mujer en edad 


\section{Diagnóstico y manejo del síndrome de ovario poliquístico}

Vol. 3, núm. 3., (2019)

Becquer Humberto Suarez Coba; Patricia Elisabeth Borja Tapia; Mayra Alexandra Vela

Chasiluisa; Carlos Fabián Ontaneda Tenesaca

reproductiva con hirsutismo u otras manifestaciones cutáneas de hiperandrogenismo, irregularidades menstruales y obesidad. Esta patología representa amerita de un diagnóstico oportuno, el cual debe diferenciarse de los cambios fisiológicos propios de la edad y de otros trastornos hiperandrogénicos que requieren de una terapia específica, debido a que se asocia a riesgos reproductivos, oncológicos y metabólicos, siendo necesario que su tratamiento se asocie con la corrección de las alteraciones metabólica. (Pereira, 2016).

Tipo de investigación

Dentro de toda práctica investigativa, se precisan acciones de carácter metodológico mediante las cuales, se logra conocer y proyectar los eventos posibles que la determinan, así como las características que hacen del acto científico un proceso interactivo ajustado a una realidad posible de ser interpretada. En este sentido, se puede decir, que la presente investigación corresponde al tipo documental, definido por (Dávila, 2012), "se ocupa del estudio de problemas planteados a nivel teórico, la información requerida para abordarlos se encuentra básicamente en materiales impresos, audiovisuales y /o electrónicos”. (p.41).

En consideración a esta definición, la orientación metodológica permitió la oportunidad de cumplir con una serie de actividades inherentes a la revisión y lectura de diversos documentos donde se encontraron ideas explicitas relacionadas con los tópicos encargados de identificar a cada característica insertada en el estudio. Por lo tanto, se realizaron continuas interpretaciones con el claro propósito de revisar aquellas apreciaciones o investigaciones propuestas por diferentes investigadores, para luego dar la respectiva argumentación a los planteamientos, en función a las necesidades encontradas en la indagación. 


\section{Diagnóstico y manejo del síndrome de ovario poliquístico}

Vol. 3, núm. 3., (2019)

Becquer Humberto Suarez Coba; Patricia Elisabeth Borja Tapia; Mayra Alexandra Vela Chasiluisa; Carlos Fabián Ontaneda Tenesaca

\section{Fuentes documentales}

El análisis correspondiente a las características que predomina en el tema seleccionado, llevan a incluir diferentes fuentes documentales encargadas de darle el respectivo apoyo y en ese sentido cumplir con la valoración de los hechos a fin de generar nuevos criterios que sirven de referencia a otros procesos investigativos. Para (Arias, 2010), las fuentes documentales incorporadas en la investigación documental o bibliográfica, "representa la suma de materiales sistemáticos que son revisados en forma rigurosa y profunda para llegar a un análisis del fenómeno". (p.41). Por lo tanto, se procedió a cumplir con la realización de una lectura previa determinada por encontrar aquellos aspectos estrechamente vinculados con la "DIAGNÓSTICO Y MANEJO DEL SÍNDROME DE OVARIO POLIQUÍSTICO” para luego explicar mediante un desarrollo las respectivas apreciaciones generales de importancia.

\section{Técnicas para la Recolección de la Información}

La conducción de la investigación para ser realizada en función a las particularidades que determinan a los estudios documentales, tiene como fin el desarrollo de un conjunto de acciones encargadas de llevar a la selección de técnicas estrechamente vinculadas con las características del estudio. En tal sentido, (Arias Ob cit) refiere, que es "una técnica particular para aportar ayuda a los procedimientos de selección de las ideas primarias y secundarias”. (p. 71).

Por ello, se procedió a la utilización del subrayado, resúmenes, fichaje, como parte básica para la revisión y selección de los documentos que presentan el contenido teórico. Es decir, que mediante su aplicación de estas técnicas se pudo llegar a recoger informaciones en cuanto a la revisión bibliográfica de los diversos elementos encargados de orientar el proceso de 


\section{Diagnóstico y manejo del síndrome de ovario poliquístico}

Vol. 3, núm. 3., (2019)

Becquer Humberto Suarez Coba; Patricia Elisabeth Borja Tapia; Mayra Alexandra Vela

Chasiluisa; Carlos Fabián Ontaneda Tenesaca

investigación. Tal como lo expresa, (Arias Ob cit) "las técnicas documentales proporcionan las herramientas esenciales y determinantes para responder a los objetivos formulados y llegar a resultados efectivos" (p. 58). Es decir, para responder con eficiencia a las necesidades investigativas, se introdujeron como técnica de recolección el método inductivo, que hizo posible llevar a cabo una valoración de los hechos de forma particular para llegar a la explicación desde una visión general.

Asimismo, se emplearon las técnicas de análisis de información para la realización de la investigación que fue ejecutada bajo la dinámica de aplicar diversos elementos encargados de determinar el camino a recorrer por el estudio, según, (Arias, Ob cit) las técnicas de procesamiento de datos en los estudios documentales "son las encargadas de ofrecer al investigador la visión o pasos que debe cumplir durante su ejercicio, cada una de ellas debe estar en correspondencia con el nivel a emplear" (p. 123). Esto indica, que para llevar a cabo el procesamiento de los datos obtenidos, es necesario establecer las técnicas que serán seleccionadas, destacándose en este caso, de manera particular: fichas de resumen, textual, registros descriptivos entre otros, los mismos se deben ajustar al nivel que ha sido seleccionado.

\section{Resultados.}

Síndrome de ovario poliquístico

El síndrome de ovario poliquístico (SOP) es un trastorno que se caracteriza por la aparición de varios quistes dentro del ovario produciéndose una alteración que incrementa los niveles de hormonas masculinas (andrógenos) y provoca la aparición de acné, caída de cabello e hirsutismo (excesiva vellosidad) sin saber las verdaderas causas. (De Santis, 2017). Hasta el 


\section{Diagnóstico y manejo del síndrome de ovario poliquístico}

Vol. 3, núm. 3., (2019)

Becquer Humberto Suarez Coba; Patricia Elisabeth Borja Tapia; Mayra Alexandra Vela Chasiluisa; Carlos Fabián Ontaneda Tenesaca

momento se desconoce la causa exacta del SOP, pero se sabe que los antecedentes familiares son un factor de riesgo importante para su aparición ya que la mayoría de las pacientes son hijas de mujeres que han tenido este trastorno. Cuando se produce una alteración hormonal los períodos menstruales se tornan irregulares, escasos o pueden desaparecer, además la falta de ovulación genera infertilidad. Más de la mitad de las mujeres con este problema de salud tiene problemas para lograr embarazarse

\section{Fisiopatología}

En la compleja fisiopatología del síndrome de ovario poliquístico, destacan al menos tres tipos de alteraciones interrelacionadas entre sí: una disfunción neuroendocrina (hipersecreción de LH), un trastorno metabólico (resistencia insulínica e hiperinsulinemia) y una disfunción de la esteroidogénesis y de la foliculogénesis ovárica. Esta patología representa una disfunción euroendocrina y caracteriza por un aumento de la secreción de LH y una secreción de FSH normal o disminuida. (De Santis, 2017). En estas pacientes se ha observado un aumento de la amplitud y frecuencia de los pulsos de LH, lo que reflejaría un aumento de los pulsos del factor liberador de gonadotrofinas $(\mathrm{GnRH})$. No se han identificado alteraciones en neurotransmisores específicos que expliquen este trastorno y las evidencias actuales sugieren que se trataría probablemente de una disfunción hipotalámica secundaria a los niveles elevados de andrógenos e insulina

\section{Disfunción Metabólica}

Está representada principalmente por una RI periférica que se expresa por una hipersecreción de insulina. Esta a su vez, promueve una mayor secreción de andrógenos por el 


\section{Diagnóstico y manejo del síndrome de ovario poliquístico}

Vol. 3, núm. 3., (2019)

Becquer Humberto Suarez Coba; Patricia Elisabeth Borja Tapia; Mayra Alexandra Vela

Chasiluisa; Carlos Fabián Ontaneda Tenesaca

ovario y las suprarrenales; estimula la secreción de LH y además disminuye la síntesis hepática de la SHBG (globulina trasportadora de hormonas sexuales) con lo cual aumenta la fracción libre y actividad biológica de los andrógenos. De acuerdo a estudiOS, la hipersecreción de insulina se manifiesta desde la pubertad temprana y precede al hiperandrogenismo bioquímico. El mecanismo por el cual se genera una resistencia insulínica en el síndrome de ovario poliquístico no está claro. En estas pacientes se ha establecido, que no habría una alteración del receptor de insulina ni del número de ellos sino que de los eventos post-receptor en cualquier punto de la señalización insulínica. (De Santis, 2017).

En el síndrome de ovario poliquístico semejante a lo descrito en la diabetes 2, la RI precede a la disminución de la tolerancia a la glucosa. No todas las pacientes con SOP y RI desarrollan una intolerancia a la glucosa y una diabetes tipo 2, por lo que se ha sugerido que en estos casos, debe coexistir una disfunción de la célula â-pancreática la cual podría ser condicionada por el mismo defecto que genera la resistencia insulínica o por otros factores. En la minoría de los casos (20-30 \%), el SOP puede manifestarse sin resistencia insulínica, lo que se debería a que por ser una enfermedad multigénica compleja no siempre se heredan conjuntamente genes asociados a RI con genes asociados a la disfunción reproductiva. (De Santis, 2017). Estas disfunciones se definen de la siguiente forma:

\section{Disfunción de la esteroidogénesis ovárica/suprarrenal}

Es un pilar fundamental en este síndrome y se caracteriza por una alteración de la biosíntesis de los andrógenos, la cual tanto en el ovario como en la suprarrenal está determinada por la actividad de una enzima denominada citocromo P450c17. En pacientes con síndrome de 


\section{Diagnóstico y manejo del síndrome de ovario poliquístico}

Vol. 3, núm. 3., (2019)

Becquer Humberto Suarez Coba; Patricia Elisabeth Borja Tapia; Mayra Alexandra Vela Chasiluisa; Carlos Fabián Ontaneda Tenesaca

ovario poliquístico la actividad de esta enzima está aumentada, lo que lleva a una mayor producción de andrógenos ováricos y adrenales. El aumento de los andrógenos intraováricos, alteran el desarrollo de los folículos y la ovulación. (De Santis, 2017) El hiperandrogenismo adrenal funcional está presente en alrededor del 50\% de las mujeres con síndrome de ovario poliquístico, y se expresa por una elevación moderada de DHEAS. Se ha propuesto que la disfunción de esta enzima (P450c17) sería exclusiva del síndrome de ovario poliquístico pudiendo ser un evento primario o secundario al exceso de LH y/o insulina; la cual potenciaría esta disfunción (18). Además, cabe destacar que el tejido adiposo juega un papel preponderante en la fisiopatología del SOP ya que tiene una función esteroidogénica intrínseca y es un tejido blanco para los andrógenos

\section{Disfunción de la foliculogénesis}

Se ha podido establecer, mediante estudios ultrasonográficos y biopsias ováricas, que las pacientes con SOP presentan un pool de folículos en crecimiento 2 a 3 veces superior que las mujeres sanas. La histología del síndrome de ovario poliquístico se caracteriza por un aumento de folículos preantrales y antrales pequeños y un mayor reclutamiento folicular. Esta situación se acompaña además de una detención del proceso de selección folicular, lo que explica la ausencia de ovulación. Por lo tanto, en el síndrome de ovario poliquístico habría mayor reclutamiento y una menor selección, lo que mantiene un aumento del pool de folículos en crecimiento productores de andrógenos. (De Santis, 2017)

En los últimos años se ha propuesto que la Hormona Antimülleriana (AMH) podría ser utilizada como un marcador sérico de la reserva folicular. La AMH es una glicoproteína dimérica 


\section{Diagnóstico y manejo del síndrome de ovario poliquístico}

Vol. 3, núm. 3., (2019)

Becquer Humberto Suarez Coba; Patricia Elisabeth Borja Tapia; Mayra Alexandra Vela

Chasiluisa; Carlos Fabián Ontaneda Tenesaca

miembro de la superfamilia TGFâ, producida exclusivamente por las células de la granulosa en la mujer. Su concentración es independiente de las gonadotrofinas y por lo tanto refleja la reserva ovárica en cualquier momento de la vida de la mujer. Además, recientemente hemos podido establecer que las hijas de mujeres con síndrome de ovario poliquístico tienen niveles significativamente mayores de AMH desde la infancia temprana (2 a 3 meses de vida) hasta la peripubertad, lo que sugiere que estas niñas nacen con una masa de folículos aumentada, lo que podría constituir un eslabón para el desarrollo ulterior de SOP. (De Santis, 2017)

\section{Etiopatogenia}

El síndrome de ovario poliquístico tiene una base genética y puede comprometer a otros miembros de la familia. Tanto las hijas como las hermanas así como los hijos y los hermanos del caso índice pueden manifestar algún rasgo fenotípico de este síndrome, que es considerado en la actualidad una enfermedad familiar multigénica compleja que afecta además al varón. Entre los factores ambientales destacan la obesidad y los eventos que ocurren en la vida intrauterina (hiperandrogenismo, diabetes gestacional y sobrepeso de la madre durante el embarazo). Por lo tanto, es de suma importancia el manejo adecuado de la embarazada, ya que estudios epidemiológicos y clínicos sugieren una relación entre el ambiente prenatal y el riesgo de desarrollar enfermedades metabólicas durante la edad adulta. (De Santis, 2017).

\section{Diagnóstico del ovario poliquístico}

\section{Criterios Diagnósticos}




\section{Diagnóstico y manejo del síndrome de ovario poliquístico}

Vol. 3, núm. 3., (2019)

Becquer Humberto Suarez Coba; Patricia Elisabeth Borja Tapia; Mayra Alexandra Vela Chasiluisa; Carlos Fabián Ontaneda Tenesaca

El síndrome de ovario poliquístico (SOP), también denominado hiperandrogenismo ovárico funcional o anovulación crónica hiperandrogénica, es una disfunción endocrinometabólica de alta prevalencia. Es la causa más común de hiperandrogenismo con una incidencia de un 3\% tanto en mujeres adolescentes como adultas. Se estima, que está presente en el $75 \%$ de las mujeres hirsutas y en el $10 \%$ de las mujeres premenopaúsicas. En los últimos años se ha podido establecer, que este trastorno no sólo está limitado a la mujer en etapa reproductiva sino que puede manifestarse desde el período prepuberal y quizás antes. (Balledero, 2015)

Su etiología es incierta y se manifiesta por síntomas y signos variados que afectan a cada mujer en forma particular. Entre ellos destacan las irregularidades menstruales, las manifestaciones cutáneas del hiperandrogenismo, la obesidad, la infertilidad, la resistencia insulínica (RI) y el aspecto poliquístico de los ovarios en la ultrasonografía. El diagnóstico de SOP es particularmente importante debido a que identifica riesgos metabólicos y cardiovasculares así como el potencial reproductivo de estas pacientes. Lo anterior implica que las pacientes en las cuales se establece el diagnóstico de SOP deben ser informadas y educadas respecto a su patología, deben ser diagnosticadas y tratadas oportunamente y deben ser controladas en forma prolongada. (Balledero, 2015).

Tabla $\mathbf{N}^{\circ}$ 1. Parámetros utilizados para la evaluación de los distintos criterios de SOP 


\section{Diagnóstico y manejo del síndrome de ovario poliquístico}

Vol. 3, núm. 3., (2019)

Becquer Humberto Suarez Coba; Patricia Elisabeth Borja Tapia; Mayra Alexandra Vela

Chasiluisa; Carlos Fabián Ontaneda Tenesaca

\begin{tabular}{|lll|}
\hline \multicolumn{1}{|c|}{ Parámetros } & \multicolumn{1}{c|}{ Categoría } & \multicolumn{1}{c|}{ Descripción } \\
\hline Estado androgénico & $\begin{array}{l}\text { Hiperandrogenismo } \\
\text { clínico }\end{array}$ & $\begin{array}{l}\text { El hiperandrogenismo clínico puede incluir hirsutismo (vello excesivo } \\
\text { terminal que aparece en patrón masculino), acné o alopecia androgenética }\end{array}$ \\
\cline { 2 - 4 } & $\begin{array}{l}\text { Hiperandrogenismo } \\
\text { bioquímico }\end{array}$ & $\begin{array}{l}\text { Andrógenos elevados en sangre, referido a aumento total, biodisponibilidad } \\
\text { o niveles libres de testosterona en sangre, aunque dada su variabilidad en } \\
\text { sangre, es difícil definir un nivel especifico para el diagnóstico. }\end{array}$ \\
\hline $\begin{array}{ll}\text { Historia menstrual } \\
\text { (ciclos) }\end{array}$ & $\begin{array}{l}\text { Anovulatorio u } \\
\text { Oligo-ovulatorio* }\end{array}$ & $\begin{array}{l}\text { Anovulación puede manifestarse como sangrados frecuentes a intervalos } \\
\text { inferiores a 21 dias o sangrados infrecuentes mayores a 35 dias. A veces la } \\
\text { menstruación puede ser anovulatoria pese a incurrir en ciclo normal (25-35 } \\
\text { días). La objetivación de progesterona a mitad del ciclo puede ayudar con el } \\
\text { diagnóstico si las menstruaciones sugieren ovulaciones normales. }\end{array}$ \\
& & $\begin{array}{l}\text { La morfología de ovario poliquístico se define como 12 o más folículos de } \\
\text { 2-9 mm de diámetro o aumento de volumen ovárico }>10 \text { ml sin necesidad } \\
\text { de folículo dominante en cualquiera de los ovarios. }\end{array}$ \\
\hline Hallazgos ováricos & Tamaño/morfología \\
& ovárica en ecografia & \\
& &
\end{tabular}

Fuente: (Balledero, 2015).

Siguiendo las bases de lo señalado anteriormente, se establece que existen diferentes parámetros que son utilizados en el diagnóstico del Síndrome de Ovarios Poliquísticos; entre ellos se encuentran:

Consenso de NIH, 1990

No fue recién hasta 1990 en que se reunió un grupo de expertos y confeccionaron la primera definición clínica de SOP. Esta fue realizada por la NIH en Bethesda, Maryland en Estados Unidos. En este consenso, SOP fue definido, en orden de importancia como: 1) hiperandrogenismo clínico o de laboratorio junto con; 2) anovulación crónica; y 3) exclusión de otros síndromes. La morfología de los ovarios no era parte de los criterios diagnósticos, sólo refería que podían sugerirlos. (Balledero, 2015). 


\section{Diagnóstico y manejo del síndrome de ovario poliquístico}

Vol. 3, núm. 3., (2019)

Becquer Humberto Suarez Coba; Patricia Elisabeth Borja Tapia; Mayra Alexandra Vela Chasiluisa; Carlos Fabián Ontaneda Tenesaca

De estos criterios no fue especificado qué significaba y cómo se medía el hiperandrogenismo clínico o de laboratorio. Tampoco, fue establecido, cuáles andrógenos medir, ni que características clínicas serían diagnósticas, debido a que los mismos se basaron en su mayoría en opiniones y no en evidencia científica. Con el tiempo cada vez se hacía más evidente que la expresión clínica de este síndrome era más amplia y variada que la presentada en el consenso de la NIH, y que la morfología del ovario era consistente en la mayoría de las mujeres con SOP. (Balledero, 2015).

Consenso de ROTTERDAM, 2003

Pasaron 15 años desde este primer consenso cuando surge un segundo. Este es el creado en el año 2003 en Rotterdam, por la ESHRE (European Society for Human Reproduction and Embriology) y la ASRM (American Society for Reproductive Medicine).Se consideraron tres criterios diagnósticos, debiéndose cumplir dos de ellos para realizar el diagnóstico. Los criterios eran: 1) oligo o anovulación; 2) hiperandrogenismo clínico y/o de laboratorio; y 3) ovarios poliquísticos. (Balledero, 2015).

Gráfica $\mathbf{N}^{\circ}$ 1. Diagnóstico de SOP según criterios, en mujeres en edad reproductiva atendidas en el servicio de ginecología del Hospital Alemán Nicaragüense entre Enero del 2009 y Diciembre del 2014. 


\section{Diagnóstico y manejo del síndrome de ovario poliquístico}

Vol. 3, núm. 3., (2019)

Becquer Humberto Suarez Coba; Patricia Elisabeth Borja Tapia; Mayra Alexandra Vela

Chasiluisa; Carlos Fabián Ontaneda Tenesaca

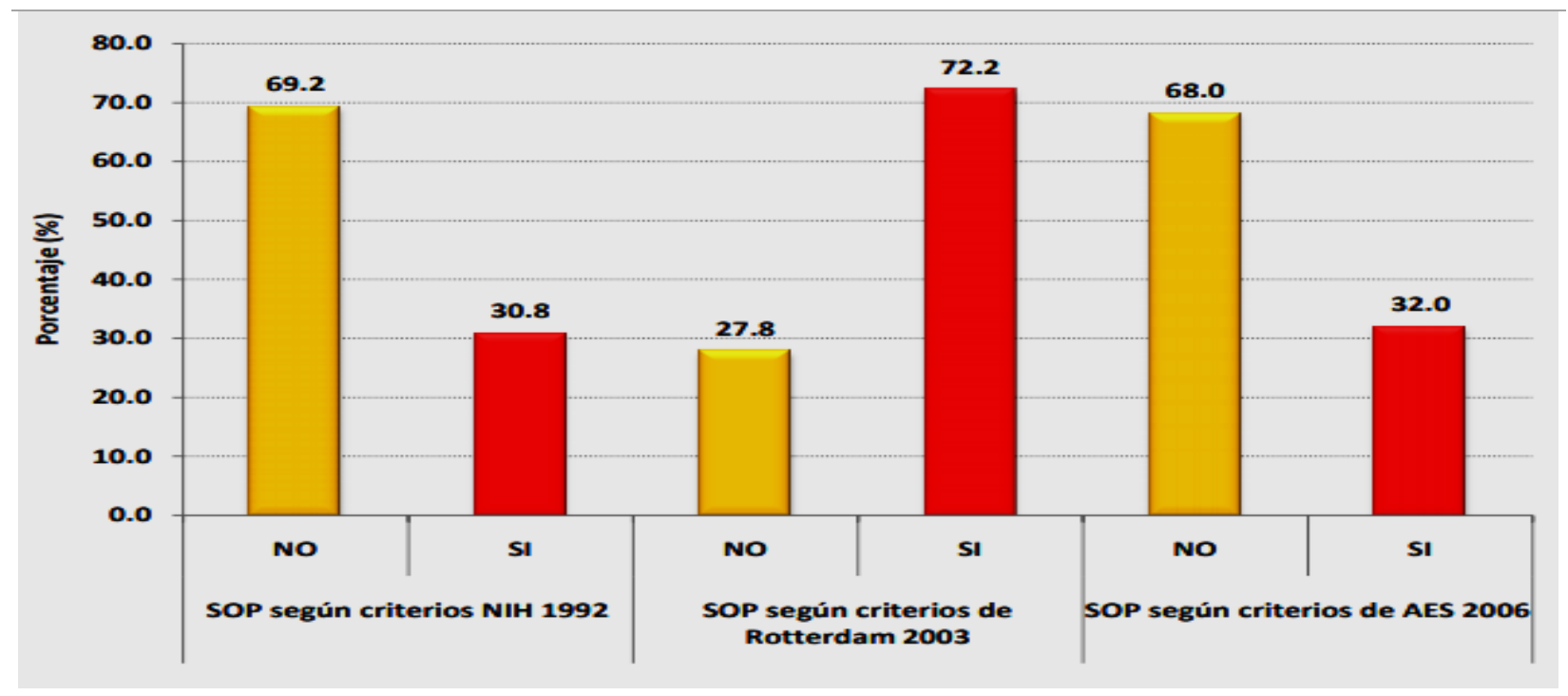

Fuente: (Balledero, 2015).

En este consenso se reconoció la existencia de fenotipos de SOP con ciclos regulares, y fenotipos sin hiperandrogenismo. Se incluyó la morfología ecográfica como uno de los criterios diagnósticos; con ello se definen 4 fenotipos diferentes. Debían al igual que en NIH, excluirse otras patologías que provocaran hiperandrogenismo, entre las cuales menciona hiperplasia suprarrenal congénita, síndrome de Cushing y tumores secretores de andrógenos. Además, indica que se debe descartar hipogonadismo hipogonadotrópico, hiperprolactinemia

Tabla N². Fenotipos del SOP según consenso ROTTERDAM 2003 


\section{Diagnóstico y manejo del síndrome de ovario poliquístico}

Vol. 3, núm. 3., (2019)

Becquer Humberto Suarez Coba; Patricia Elisabeth Borja Tapia; Mayra Alexandra Vela Chasiluisa; Carlos Fabián Ontaneda Tenesaca

\begin{tabular}{|ll|}
\hline Fenotipo & Descripción \\
\hline I & HA más disfunción ovulatoria y ovario de morfología PQ en US \\
\hline II & HA más disfunción ovulatoria \\
\hline III & HA más ovario de morfología PQ en US \\
\hline IV & Disfunción ovulatoria más ovario de morfología PQ en US \\
\hline HA =hiperandrogenismo (HA) clínico y/o bioquímico; \\
PQ = poliquística; \\
US =ultrasonografia \\
\hline
\end{tabular}

Fuente: (Balledero, 2015).

Los subfenotipos basados en los criterios de Rotterdam son:

- Subfenotipo A: Oligo-ovulación, hiperandrogenismo clínico o de laboratorio, eco compatible con SOP.

- Subfenotipo B: Oligo-ovulación, hiperandrogenismo clínico o de laboratorio.

- Subfenotipo C: Hiperandrogenismo clínico o de laboratorio, eco compatible con SOP.

- Subfenotipo D: Oligo-ovulación, eco compatible con SOP.14

Los elementos clínicos más importantes a considerar son: hirsutismo, alteraciones menstruales (amenorrea, oligomenorrea), acné, obesidad, complicaciones en el embarazo (aborto recurrente, diabetes gestacional, preeclampsia), aumento del riesgo cardiovascular, hiperplasia y cáncer de endometrio, infertilidad, hiperprolactinemia, alopecia, acantosis nigricans, trastornos del sueño y síntomas neuropsiquiátricos, no existiendo elementos médicos específicos del SOP. Una correcta historia clínica, la exploración física, algunas determinaciones de laboratorio y la 


\section{Diagnóstico y manejo del síndrome de ovario poliquístico}

Vol. 3, núm. 3., (2019)

Becquer Humberto Suarez Coba; Patricia Elisabeth Borja Tapia; Mayra Alexandra Vela

Chasiluisa; Carlos Fabián Ontaneda Tenesaca

ecografía, serán las bases para poder aplicar los vigentes criterios diagnósticos de Rotterdam y excluir otras enfermedades. (Balledero, 2015)

Consenso de la AES (2006)

En el año 2006 la Sociedad de Exceso de Andrógenos (AES) realizó una declaración acerca de los criterios para diagnosticar SOP, describiendo como característica fundamental que el SOP es un síndrome predominantemente hiperandrogénico. Además se consideró que SOP era definido por todos aquellos fenotipos que aumentaban potencialmente el riesgo de insulinoresistencia y alteraciones metabólicas. Con ello sugieren que el exceso de andrógenos se incorpore como criterio diagnóstico obligatorio, ya que el fenotipo descrito por Rotterdam que no tenía esta característica, no ha demostrado tener mayor riesgo metabólico. En el fenotipo con hiperandrogenismo pero ovulatorio, se mantiene elevado el riesgo con respecto a la población general, pero es menor que el fenotipo con hiperandrogenismo y oligoanovulación. (Balledero, 2015)

Tabla $\mathbf{N}^{\circ}$ 3. Fenotipos de síndrome ovario poliquístico según AES 2006 


\section{Diagnóstico y manejo del síndrome de ovario poliquístico}

Vol. 3, núm. 3., (2019)

Becquer Humberto Suarez Coba; Patricia Elisabeth Borja Tapia; Mayra Alexandra Vela Chasiluisa; Carlos Fabián Ontaneda Tenesaca

\begin{tabular}{|ll|}
\hline Fenotipo & Descripción \\
\hline I & HA más disfunción ovulatoria y ovario de morfología PQ en US \\
\hline II & HA más disfunción ovulatoria \\
\hline III & HA más ovario de morfología PQ en US \\
\hline HA =hiperandrogenismo (HA) clínico y/o bioquímico; \\
PQ =poliquística; \\
US =ultrasonografia
\end{tabular}

Fuente: (Balledero, 2015)

Tabla $\mathbf{N}^{\circ}$ 4. Diagnóstico de SOP según criterios, en mujeres en edad reproductiva atendidas en el servicio de ginecología del Hospital Alemán Nicaragüense entre Enero del 2009 y Diciembre del 2014.

\begin{tabular}{|llcc|}
\hline $\mathbf{n}$ & & $\mathbf{n}$ & $\%$ \\
\hline Criterios NIH 1992 & & 169 & 100.0 \\
\cline { 2 - 4 } & SO & 117 & 69.2 \\
\hline Criterios de Rotterdam 2003 & NO & $\mathbf{5 2}$ & $\mathbf{3 0 . 8}$ \\
\cline { 2 - 4 } & SI & 47 & 27.8 \\
\hline AES 2006* & NO & 122 & 72.2 \\
\cline { 2 - 4 } & SI & 115 & 68.0 \\
\hline
\end{tabular}

Fuente: (Corett, 2015).

El consenso AES 2006 propone la medición del hirsutismo mediante una escala o Ferriman-Gallwey, junto con un punto de corte para definir hiperandrogenismo (puntaje para 


\section{Diagnóstico y manejo del síndrome de ovario poliquístico}

Vol. 3, núm. 3., (2019)

Becquer Humberto Suarez Coba; Patricia Elisabeth Borja Tapia; Mayra Alexandra Vela

Chasiluisa; Carlos Fabián Ontaneda Tenesaca

percentil mayor a 95, que corresponde entre 6 y 8 según la población). Tomando en cuenta esta recomendación, según la evidencia reportada en los expedientes clínicos de las pacientes en estudio la proporción de SOP usando los criterios AES 2006 sería de 7\%. (Corett, 2015)

Así definen criterios diagnósticos, consistentes en 1) hiperandogenismo clínico y/o laboratorio; 2) trastornos menstruales y/u ovarios de morfología poliquística; 3) exclusión de otros desórdenes. Se debe cumplir cada punto por separado. En este caso, se definen 3 diferentes fenotipos. Para hiperandrogenismo clínico mantiene al hirsutismo como manifestación central, dando como referencia que según los criterios de NIH aproximadamente $60 \%$ de las pacientes con SOP lo presentarían. Se propone por primera vez en un consenso una escala de medición de éste, el puntaje de Ferriman-Gallwey, junto con un punto de corte para definir hiperandrogenismo (puntaje para percentil mayor a 95, que corresponde entre 6 y 8 según la población). No obstante, se reconoce que las escalas usadas para esta medición son subjetivas. (Corett, 2015).

Gráfico $\mathbf{N}^{\circ}$ 2. Frecuencia según tipo de componente del SOP en mujeres en edad reproductiva atendidas en el servicio de ginecología del Hospital Alemán Nicaragüense entre Enero del 2009 y Diciembre del 2014 


\section{Diagnóstico y manejo del síndrome de ovario poliquístico}

Vol. 3, núm. 3., (2019)

Becquer Humberto Suarez Coba; Patricia Elisabeth Borja Tapia; Mayra Alexandra Vela Chasiluisa; Carlos Fabián Ontaneda Tenesaca

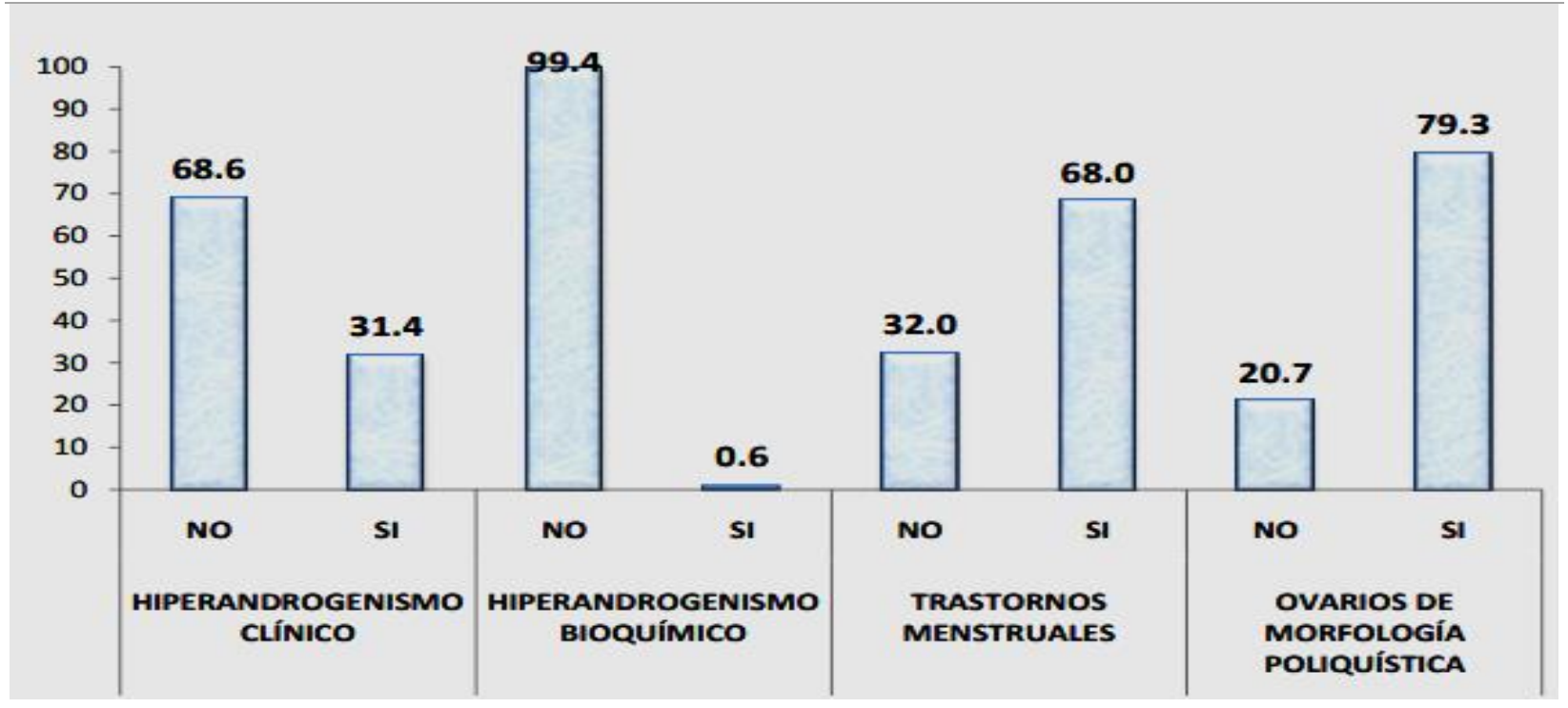

Fuente: (Corett, 2015)

De igual manera, los criterios manejados para desarrollar diagnósticos son clasificados por especialistas de la siguiente forma:

Diagnóstico en Adultos: el síndrome de ovario poliquístico es un síndrome de etiología compleja, y escasamente comprendida. Para el diagnóstico en adultos se realiza cumpliendo dos de los tres criterios: hiperandrogenismo, ovarios poliquísticos (objetivados mediante técnica ecográfica) o disfunción ovárica. Se deben excluir las entidades que en su desarrollo natural de enfermedad presenten clínica que pueda solaparse, como las afecciones tiroideas, hiperprolactinemia e hiperplasia suprarrenal congénita (mayormente déficit primario de la 21 hidroxilasa). Los criterios más utilizados para el diagnóstico son los de Rotterdam, aunque es frecuente usar otros criterios diagnósticos. (Corett, 2015).

Diagnóstico en Adolescencia: la mayoría de guías europeas recomiendan realizar el diagnóstico de síndrome de ovario poliquístico ante la presencia de clínica sugestiva y/o 


\section{Diagnóstico y manejo del síndrome de ovario poliquístico}

Vol. 3, núm. 3., (2019)

Becquer Humberto Suarez Coba; Patricia Elisabeth Borja Tapia; Mayra Alexandra Vela

Chasiluisa; Carlos Fabián Ontaneda Tenesaca

exámenes analíticos compatibles. El morfotipo de SOP y los síntomas anovulatorios no son suficientes para realizar el diagnóstico, dado que dichos síntomas son habituales durante la maduración reproductiva. Tampoco se ha demostrado que el criterio ecográfico de los criterios de Rotterdam no se puede extrapolar a la adolescencia al no haber sido validado en este grupo poblacional. Durante el primer año tras la menarquia hasta el $85 \%$ de los ciclos menstruales son anovulatorios, disminuyendo progresivamente hasta el $25 \%$ de ciclos anovulatorios a partir del sexto año tras la misma. Estos ciclos anovulatorios se suelen asociar a aumento de LH en plasma. (Corett, 2015)

Diagnóstico en Menopausia y Perimenopausia: aunque hay no criterios diagnósticos específicos para las mujeres en la etapa de menopausia y perimenopausia, sí que se puede realizar diagnóstico de presunción en el contexto de historia objetivada de hiperandrogenismo y oligoamenorrea durante su etapa reproductiva. La presencia de morfotipo de Síndrome de Ovario Poliquístico mediante ecografía puede sugerir dicho diagnóstico, aunque es algo inhabitual en la mujer menopáusica. Existen pocos estudios específicos en este ámbito, además del problema asociado a la disminución del tamaño del ovario, contaje folicular y niveles hormonales antiMuller que se encuentra en este tipo de pacientes. Tampoco existen niveles de referencia que nos

indiquen los niveles adecuados de testosterona en este tipo pacientes, aunque sí que debe descartarse un tumor productor de andrógeno en mujeres con niveles muy altos de testosterona. (Corett, 2015)

Diagnóstico diferencial del SOP 


\section{Diagnóstico y manejo del síndrome de ovario poliquístico}

Vol. 3, núm. 3., (2019)

Becquer Humberto Suarez Coba; Patricia Elisabeth Borja Tapia; Mayra Alexandra Vela Chasiluisa; Carlos Fabián Ontaneda Tenesaca

Al tener en cuenta los posibles diagnósticos diferenciales de esta patología, es de gran utilidad tomar en cuenta los criterios diagnósticos anteriormente mencionados y pensar en otras causas que las desencadenen.

1. Hiperandrogenismo: Los andrógenos son producidos tanto por las glándulas suprarrenales como por los ovarios, a través de un eje de retrocontrol en el que están involucradas hormonas producidas por el hipotálamo y la hipófisis. En este caso se deben tomar en cuenta las patologías a nivel central que pueden producir un exceso de hormonas que realicen un retrocontrol positivo en la producción de andrógenos, por ejemplo: la presencia de un prolactinoma, acromegalia o de disfunción hipotalámica. Además, el hiperandrogenismo puede darse por cualquier patología que involucre las glándulas suprarrenales encargadas de la producción más que todo de andrógenos débiles como la dehidroepiandrosterona (DHEA) y el sulfato de dehidroepiandrosterona (DHEAS), en este caso se debe tomar en cuenta la hiperplasia suprarrenal congénita no clásica, el síndrome de Cushing y los tumores secretores de andrógenos. (Vega, 2015)

2. Amenorrea/ Oligomenorrea: En este caso se deben tomar en cuenta todas las posibles causas de amenorrea, recordando que el SOP puede estar ligado al mismo estado de hiperandrogenismo característico. Entre las posibles causas de amenorrea/ oligomenorrea probables se pueden enumerar la falla ovárica primaria anteriormente mencionada y la presencia de hipotiroidismo. (Vega, 2015) 


\section{Diagnóstico y manejo del síndrome de ovario poliquístico}

Vol. 3, núm. 3., (2019)

Becquer Humberto Suarez Coba; Patricia Elisabeth Borja Tapia; Mayra Alexandra Vela

Chasiluisa; Carlos Fabián Ontaneda Tenesaca

3. Hiperinsulinemia: Si bien esta entidad no es considerada dentro de los criterios diagnósticos, constituye un hallazgo común en estas mujeres; por lo cual se deben tomar en cuenta los antecedentes personales y familiares de diabetes, así como los de enfermedad cardiovascular y otras causas de hiperinsulinemia como la presencia de un insulinoma y defectos genéticos en la acción de la insulina. Dentro de la evaluación de la paciente es indispensable una historia clínica detallada donde se describan los antecedentes familiares y personales así como la evolución de la enfermedad. El examen físico debe incluir la búsqueda de hiperandrogenismo clínico y de signos de hiperinsulinemia, siendo la acantosis nigricans un signo importante de señalar. (Vega, 2015)

\section{Aspectos clínicos}

El cuadro clínico es muy polimorfo y varía de acuerdo a la edad de la paciente. Por lo general las manifestaciones clínicas se inician en el período perimenárquico con la aparición de alteraciones menstruales en los dos tercios de las adolescentes principalmente oligomenorrea (sangrados con intervalos mayores de 45 días o menos de 9 sangrados al año) alternados con períodos de amenorrea secundaria (ausencia de sangrado por lo menos en tres meses consecutivos), las que cursan con buen nivel estrogénico y responden con sangrado a la administración de progesterona. (Vega, 2015).

Ocasionalmente, se presentan además episodios de metrorragia disfuncional (sangramiento excesivo fuera de ciclo) por hiperplasia endometrial. En raras oportunidades, una amenorrea primaria (ausencia de menarquia a los 16 años), una adrenarquia prematura o una pubertad precoz pueden ser el debut del cuadro clínico. En forma excepcional, las pacientes 


\section{Diagnóstico y manejo del síndrome de ovario poliquístico}

Vol. 3, núm. 3., (2019)

Becquer Humberto Suarez Coba; Patricia Elisabeth Borja Tapia; Mayra Alexandra Vela Chasiluisa; Carlos Fabián Ontaneda Tenesaca

pueden presentar una eumenorrea. (Vega, 2015). Los trastornos menstruales se asocian frecuentemente a la obesidad por lo general de tipo androide y a manifestaciones del hiperandrogenismo siendo el hirsutismo la manifestación más clásica. El hirsutismo por lo general es discreto o moderado (entre 8 y 15 puntos en la escala de Ferriman y Gallowey), se presenta en dos tercios de las pacientes, aparece después de la pubertad y progresa lentamente o se detiene alcanzada la madurez sexual. (Vega, 2015)

La virilización es rara y su presencia debe hacer sospechar otras etiologías como un tumor secretor de andrógenos o una hiperplasia adrenal congénita. Entre las manifestaciones cutáneas del hiperandrogenismo, sólo el hirsutismo, el acné y la alopecía han sido aceptadas por ambos consensos como criterio diagnóstico de hiperandrogenismo. La obesidad está presente en alrededor de la mitad de las pacientes y es típicamente de tipo androide o "forma de manzana" (índice cintura/cadera > 0.85); por lo general se inicia en la niñez y se acentúa en la pubertad. En las pacientes obesas y/o hiperinsulinémicas puede observarse acantosis nigricans que es un marcador cutáneo de resistencia insulínica, la que se presenta como una pigmentación verrucosa de color pardo oscuro que suele observarse en las zonas de pliegues. (Vega, 2015)

De igual forma, estas pacientes pueden adquirir un aspecto cushingoideo (obesidad central, dorso de búfalo y aumento de la grasa supraclavicular semejante a lo que se observa en el Cushing, pero sin atrofia muscular) o acromegaloideo (aumento del grosor de los rasgos faciales por efecto trófico de la insulina, pero sin prognatismo) que muchas veces obliga a descartar estas patologías. El cuadro clínico constituido no regresa espontáneamente (no se mejora, sólo se compensa). Con el tiempo van cambiando las manifestaciones fenotípicas. Durante la post- menarquía y edad reproductiva temprana predominan alteraciones 


\section{Diagnóstico y manejo del síndrome de ovario poliquístico}

Vol. 3, núm. 3., (2019)

Becquer Humberto Suarez Coba; Patricia Elisabeth Borja Tapia; Mayra Alexandra Vela

Chasiluisa; Carlos Fabián Ontaneda Tenesaca

reproductivas, mientras que durante la edad reproductiva tardía y peri-menopausia se acentúan las alteraciones metabólicas. Los riesgos a largo plazo derivan del hiperestrogenismo relativo (por falta de ovulación) el cual se asocia a cáncer endometrial y de la hiperinsulinemia crónica (diabetes 2 y síndrome metabólico). (Vega, 2015)

\section{Manejo del ovario poliquístico}

Existen tendencias terapéuticas que demuestran que, no existe ninguna prueba para diagnosticar definitivamente el síndrome de ovario poliquístico. Es probable que el médico comience con un análisis de tu historia clínica, incluso de tus períodos menstruales y cambios de peso. La exploración física consistirá en buscar signos de crecimiento excesivo del cabello, resistencia a la insulina y acné. Luego, podría recomendarle a la paciente:

1.- Examen pélvico. El médico revisa de forma visual y manual tus órganos genitales para detectar masas, bultos u otras anomalías.

Imagen $\mathbf{N}^{\circ}$ 1. Examen Pélvico 


\section{Diagnóstico y manejo del síndrome de ovario poliquístico}

Vol. 3, núm. 3., (2019)

Becquer Humberto Suarez Coba; Patricia Elisabeth Borja Tapia; Mayra Alexandra Vela Chasiluisa; Carlos Fabián Ontaneda Tenesaca

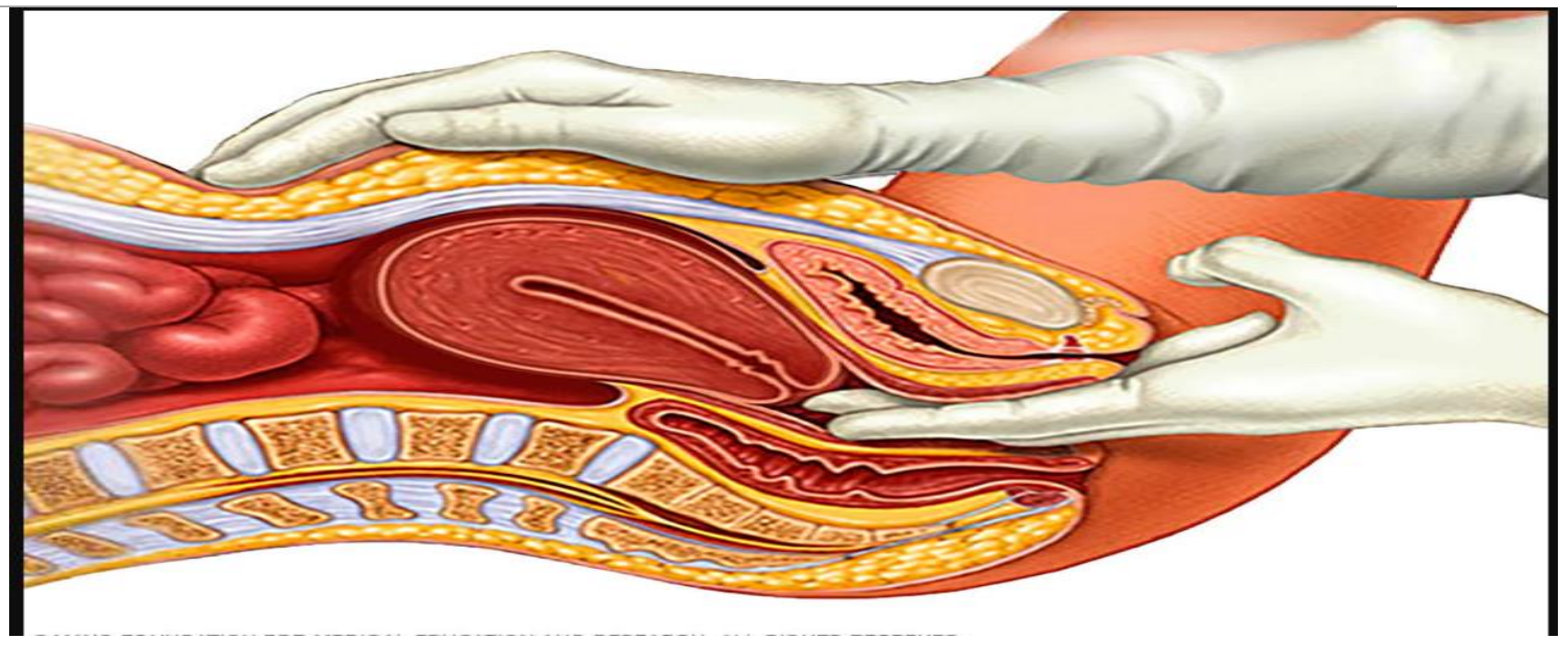

Fuente: (Vega, 2015)

2.- Análisis de sangre. Se puede analizar la sangre para medir los niveles hormonales. Esta prueba puede excluir posibles causas de anomalías menstruales o exceso de andrógenos que son similares al síndrome de ovario poliquístico. Podría realizarte un análisis de sangre adicional para medir la tolerancia a la glucosa y los niveles de colesterol y triglicéridos en ayunas.

3.- Una ecografía. El médico examina la apariencia de los ovarios y el espesor del recubrimiento del útero. Un dispositivo con forma de varilla (transductor) se coloca en la vagina (ecografía transvaginal). El transductor emite ondas sonoras que se traducen en imágenes en una pantalla de computadora.

Imagen $\mathbf{N}^{\circ}$ 2. Ecografía Transvaginal 


\section{Diagnóstico y manejo del síndrome de ovario poliquístico}

Vol. 3, núm. 3., (2019)

Becquer Humberto Suarez Coba; Patricia Elisabeth Borja Tapia; Mayra Alexandra Vela

Chasiluisa; Carlos Fabián Ontaneda Tenesaca

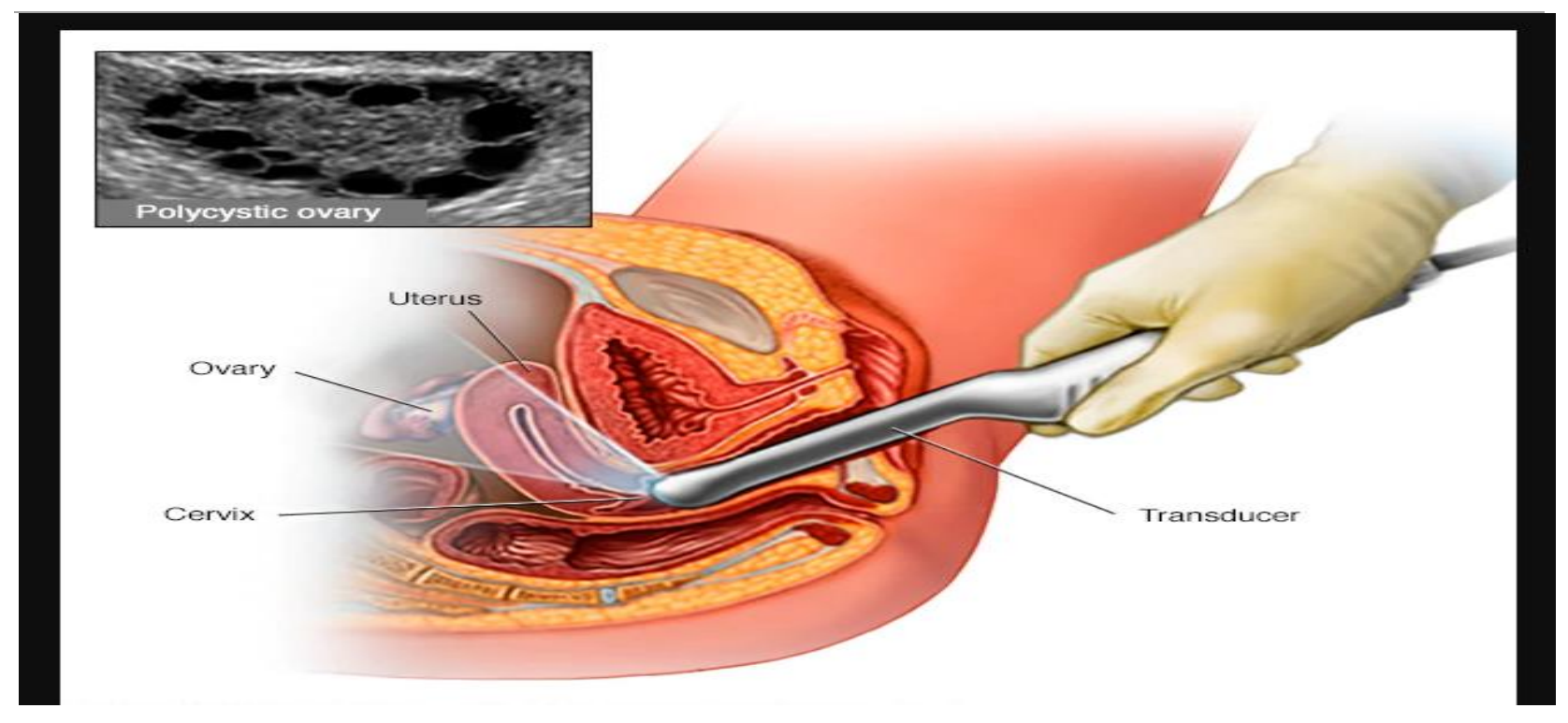

Fuente: (Vega, 2015)

Por consiguiente, es importante comprender que el manejo terapéutico del síndrome debe tener en cuenta la heterogeneidad de fenotipos con SOP. Por lo tanto, se requiere un enfoque individualizado y seguimiento a estas mujeres durante toda su vida, con énfasis en corregir el hiperandrogenismo, los trastornos menstruales, las alteraciones metabólicas asociadas (obesidad y RI) y la ovulación, en los casos en que la mujer desee embarazo.

\section{Tratamiento}

Los criterios recomendados por el Instituto Nacional de Salud y la Sociedad de Exceso de Andrógenos enfatizan la importancia del exceso de andrógenos en el diagnóstico remarcando permitiendo identificar un fenotipo asociado a mayor riesgo de complicaciones metabólicas. Entre las opciones terapéuticas podemos considerar un tratamiento no farmacológico, basado en la reducción del peso en $5 \%$ del peso inicial, la pérdida de grasa abdominal mejora el perfil 


\section{Diagnóstico y manejo del síndrome de ovario poliquístico}

Vol. 3, núm. 3., (2019)

Becquer Humberto Suarez Coba; Patricia Elisabeth Borja Tapia; Mayra Alexandra Vela Chasiluisa; Carlos Fabián Ontaneda Tenesaca

endocrino y aumenta la probabilidad de la ovulación y el embarazo. Las dietas serán bajas en calorías y en grasa, junto con un compuesto con aminoácidos, extractos y antioxidantes. Estas disminuyen la resistencia a la insulina y adicionan vitamina D para ayudar con la fertilidad e incorporar la actividad física por lo menos 90 minutos de actividad aeróbica a la semana. Si la pérdida de peso espontánea no se puede lograr con dieta y ejercicio, puede ofrecerse la cirugía bariátrica. (Vega, 2015)

El tratamiento integral también debe considerar medidas como consejería, apoyo psicológico, técnicas de meditación, relajación, educación preventiva y participación en grupos de ayuda y acompañamiento familiar. La medicina alternativa incluye muchas modalidades, como la kinesiología, la fitoterapia, la homeopatía, la reflexología, la acupresión, la acupuntura y la terapia de masaje. La acupuntura es la modalidad más común, pues parece tener beneficios para los que sufren del SO al ayudar a regular y gestionar sus períodos. Sin embargo, también se ha demostrado su utilidad para ayudar en la pérdida de peso, dolores de cabeza y mejorar los estados de ánimo.

Los métodos cosméticos tradicionales incluyen el blanqueo, depilación, afeitado, tratamiento químico y la electrólisis. El uso de fotodepilación láser de alejandrita se usa para el hirsutismo generalizado, para el tratamiento tópico y existe clorhidrato de eflornitina al 13,9 $\% .15$ Por su parte, el tratamiento farmacológico debe ser individualizado y se usará no solo pensando en la sintomatología, aspectos reproductivos, efectos indeseables, tiempo de exposición del fármaco sino también en el factor económico. El citrato de clomifeno constituye uno de los tratamientos de primera línea para la inducción de la ovulación. La metformina es un fármaco sensibilizador de insulina, esta biguanida se utiliza actualmente como un antidiabético 


\section{Diagnóstico y manejo del síndrome de ovario poliquístico}

Vol. 3, núm. 3., (2019)

Becquer Humberto Suarez Coba; Patricia Elisabeth Borja Tapia; Mayra Alexandra Vela

Chasiluisa; Carlos Fabián Ontaneda Tenesaca

oral. La metformina puede ser más eficaz en mujeres con síndrome de ovario poliquístico (PCOS), que son no obesos. (Vega, 2015)

Las tiazolidinedionas (TZD) son otra clase de fármacos sensibilizantes a la insulina que se han estudiado en mujeres con síndrome de ovario poliquístico (troglitazona, rosiglitazona y la pioglitazona). Los inhibidores selectivos de la aromatasa, como anastrozol y letrozol son prometedores nuevos agentes inductores de la ovulación. Los glucocorticoides como la prednisona y dexametasona, se han utilizado para inducir la ovulación. Sin embargo, por sus potenciales efectos adversos sobre la sensibilidad a la insulina, debe evitarse su uso prolongado. Las gonadotropinas constituyen la segunda línea posible de la terapia después de la resistencia al citrato de clomifeno, estas son las gonadotropinas exógenas. El principal inconveniente de las gonadotropinas es que provocan el desarrollo de folículos múltiples. Ello aumenta el riesgo de síndrome de hiperestimulación ovárica (SHO) y embarazos múltiples. (Vega, 2015)

La diatermia ovárica laparoscópica bilateral con electrocauterio monopolar o láser es una alternativa aceptable. La perforación laparoscópica de ovario, seguida de una estimulación correspondiente con clomifeno en asociación con la metformina es un método eficaz de tratamiento para los pacientes con infertilidad de tipo anovulatoria. En las mujeres que no desean concebir, pueden ser tratadas con píldoras anticonceptivas orales (ACO). La elección de anticonceptivo oral es importante. Deben contener etinilestradiol en dosis de $15 \mathrm{mg}$ a $35 \mathrm{mg}$ y una consideración importante para el componente de progestina (noretindrona, desogestrel, norgestimato, acetato de ciproterona, drospirenona, dienogest). (Vega, 2015) 


\section{Diagnóstico y manejo del síndrome de ovario poliquístico}

Vol. 3, núm. 3., (2019)

Becquer Humberto Suarez Coba; Patricia Elisabeth Borja Tapia; Mayra Alexandra Vela Chasiluisa; Carlos Fabián Ontaneda Tenesaca

Entre los antiandrógenos esteroideos tenemos la espironolactona, acetato de ciproterona (CPA), y los no esteroideos son la flutamida y la finasterida. La fertilización in vitro es la última posibilidad de lograr un embarazo a término en mujeres con síndrome de ovario poliquístico es el uso de técnicas de fertilización in vitro (FIV). Estas técnicas se utilizan como último recurso cuando los tratamientos con citrato de clomifeno, gonadotropinas, y el letrozol han fracasado. (Vega, 2015)

\section{Tratamiento no Farmacológico}

Ejercicio físico y pérdida de peso: dado que el SOP se asocia a obesidad y sobrepeso en una elevada proporción de casos, se recomienda pérdida de peso, tanto aumentando el ejercicio físico como con dieta hipocalórica: Se recomienda ejercicio físico en el manejo de las complicaciones de obesidad y sobrepeso de estas pacientes. Aunque no existen estudios randomizados que justifiquen esta práctica, su uso en combinación con el resto de medidas higiénico-dietéticas mejora la pérdida de peso, reducen la incidencia de desarrollo de diabetes y mejoran el resto de factores de riesgo cardiovascular. (Vega, 2015)

Se recomienda la pérdida de peso mediante dieta restrictiva. La pérdida de peso en este caso es beneficiosa para las disregulaciones metabólicas y de fertilidad, aunque en las mujeres en normopeso no se han encontrado mejorías significativas que justifique este tipo de medidas. No parece haber diferencias entre distintos tipos de dieta en términos de resultados antropométricos, pero las dietas con bajo índice glucémico podrían ser ligeramente superiores a otras en mejora de la fertilidad ( $\mathrm{Si}$ bien esta mejora se observa con cualquier pérdida de peso), así como en la mejoría de parámetros metabólicos de resistencia a la insulina. (Vega, 2015). 


\section{Diagnóstico y manejo del síndrome de ovario poliquístico}

Vol. 3, núm. 3., (2019)

Becquer Humberto Suarez Coba; Patricia Elisabeth Borja Tapia; Mayra Alexandra Vela

Chasiluisa; Carlos Fabián Ontaneda Tenesaca

(En este último caso, de forma similar a la dieta hiperprotéica y a la baja en hidratos de carbono versus hipocalórica estándar); la pérdida de peso asimismo parece mejorar la calidad de vida, autoestima y la depresión en las escalas psicológicas. No obstante, la calidad de la evidencia disponible no permite recomendar un tipo de dieta concreto sobre otro.

\section{Tratamiento Farmacológico}

Anticoncepción hormonal: se recomienda métodos de anticoncepción hormonal (pastilla, anillo vaginal o parche) como tratamiento de primera línea para el manejo del acné/hirsutismo y las disregulaciones menstruales. Se recomienda también el cribado de contraindicaciones de anticoncepción hormonal, aunque no existe una indicación con mayor grado de recomendación sobre las demás para el tratamiento del SOP, pero el riesgo-beneficio puede ser variable entre preparados y fórmula. (Vega, 2015)

En las mujeres con síndrome de ovario poliquístico la progesterona se encuentra disminuida y la producción ovárica de andrógenos. Algunos progestágenos poseen efectos antiandrogénicos, dado que antagonizan efectos de los receptores androgénicos y/o inhiben la actividad de la -alfa-reductasa. Se recomienda el uso de ciclos largos de tratamiento para evitar el efecto rebote en estas pacientes. (Vega, 2015).

Metformina: Se recomienda evitar la metformina como primer escalón terapéutico para el tratamiento sistemático de manifestaciones cutáneas, obesidad o manejo de complicaciones del embarazo. Sin embargo su uso sí se encuentra recomendado en pacientes con tolerancia anormal 


\section{Diagnóstico y manejo del síndrome de ovario poliquístico}

Vol. 3, núm. 3., (2019)

Becquer Humberto Suarez Coba; Patricia Elisabeth Borja Tapia; Mayra Alexandra Vela Chasiluisa; Carlos Fabián Ontaneda Tenesaca

a la glucosa o diabetes mellitus tipo 2 que no respondan a medidas higiénico-dietéticas. La AACE (American Association of Clinical Endocrinologists), en su posicionamiento de 2005, recomiendan metformina como primera opción terapéutica en estos casos, y considerar en el resto como segunda opción. (Vega, 2015)

\section{Conclusiones.}

El Síndrome de Ovario Poliquístico SOP representa una enfermedad con diagnóstico de exclusión, pero la tendencia actual es investigar la hormona Antimulleriana como predictor de esta enfermedad. El tratamiento está orientado a corregir el hiperandrogenismo, los trastornos menstruales, las alteraciones metabólicas asociadas y la ovulación Por ser una disfunción endocrino-metabólica con un variado espectro de anormalidades, muchas de las cuales son sutiles, las pacientes suelen consultar en diversas especialidades donde, por lo general, se da énfasis al motivo de consulta con lo cual el diagnóstico de SOP pasa desapercibido y se pierde la visión global de lo que involucra su diagnóstico.

El abordaje diagnóstico implica ciertas dificultades en relación al momento de presentación de los síntomas y la tendencia actual es considerarlo como un diagnóstico de exclusión, empleándose los cuatros fenotipos propuestos en Rotterdam. El estudio encontró que los cambios en el estilo de vida, la reducción del peso, dieta, ejercicios, medicina alternativa, tratamientos cosméticos, prevención de factores epigenéticos, la terapia quirúrgica y farmacológica contribuyen a mejorar la calidad de vida y sintomatología en las pacientes afectadas con esta entidad nosológica. 


\section{Diagnóstico y manejo del síndrome de ovario poliquístico}

Vol. 3, núm. 3., (2019)

Becquer Humberto Suarez Coba; Patricia Elisabeth Borja Tapia; Mayra Alexandra Vela

Chasiluisa; Carlos Fabián Ontaneda Tenesaca

Es importante establecer que, el diagnóstico de SOP es particularmente importante debido a que identifica riesgos metabólicos y cardiovasculares así como el potencial reproductivo de estas pacientes. Lo anterior implica que las pacientes en las cuales se establece el diagnóstico de SOP deben ser informadas y educadas respecto a su patología, deben ser diagnosticadas y tratadas oportunamente y deben ser controladas en forma prolongada.

No obstante, se encuentran criterios terapéuticos, en los cuales, los especialistas llegan a considerar un tratamiento no farmacológico, basado en la reducción del peso en $5 \%$ del peso inicial, la pérdida de grasa abdominal mejora el perfil endocrino y aumenta la probabilidad de la ovulación y el embarazo. Las dietas serán bajas en calorías y en grasa, junto con un compuesto con aminoácidos, extractos y antioxidantes. Estas disminuyen la resistencia a la insulina y adicionan vitamina D para ayudar con la fertilidad e incorporar la actividad física por lo menos 90 minutos de actividad aeróbica a la semana. Si la pérdida de peso espontánea no se puede lograr con dieta y ejercicio, puede ofrecerse la cirugía bariátrica.

Por todo esto, de manera general se plantea un tratamiento integral, el cual debe considerar medidas como consejería, apoyo psicológico, técnicas de meditación, relajación, educación preventiva y participación en grupos de ayuda y acompañamiento familiar. La medicina alternativa incluye muchas modalidades, como la kinesiología, la fitoterapia, la homeopatía, la reflexología, la acupresión, la acupuntura y la terapia de masaje. La acupuntura es la modalidad más común, pues parece tener beneficios para los que sufren del SO al ayudar a regular y gestionar sus períodos. Sin embargo, también se ha demostrado su utilidad para ayudar en la pérdida de peso, dolores de cabeza y mejorar los estados de ánimo. 


\section{Diagnóstico y manejo del síndrome de ovario poliquístico}

Vol. 3, núm. 3., (2019)

Becquer Humberto Suarez Coba; Patricia Elisabeth Borja Tapia; Mayra Alexandra Vela Chasiluisa; Carlos Fabián Ontaneda Tenesaca

\section{Bibliografía.}

Arias, F. (2010). Paradigmas de la Investigación Científica. España: Editorial: Luces.

Balledero, P. (2015). Complicaciones en pacientes con Síndrome de Ovarios Poliquísticos. Journal of the American College of Surgeons, 21 - 45.

Calletano, B. (2016). Manejo y Tratamiento de Síndrome de Ovario Poliquístico. Revista: The Journal Endicronología. EEUU., 15 - 28.

Corett, G. (2015). Etiología y Tratamiento del Síndrome de Ovario Poliquístico. Clinical Review: The Journal Medic, 34- 54.

Dávila, N. (2012). Paradigmas de la Investigación Científica. Pereire, Colombia: Editorial: Las Brisas.

De Santis, O. (2017). Riesgo aumentado de lesiones colónicas preneoplásicas en acromegalia: estudio multicéntrico caso control. Resultados preliminares. Revista Clínica de Endicronología de Buenos Aires, Argentina, 10 - 36.

Pereira, C. (2016). Manejo Clínico de Síndrome de Ovario Poliquístico. Revista Vida y Salud de la Universidad de la Habana en Cuba, 28 - 43.

Vega, S. (2015). Tratamiento en patologías de Ovario Poliquístico. Rev Española de Endicronología de la Universidad Computense de Madrid., 8 - 11. 